\title{
L'analyse comparée d'une tâche "scolaire" mise en œuvre avec des enfants de 4 ans dans une classe enfantine et dans une crèche à Genève
}

\section{Anne-Marie Munch et Maria-Luisa Schubauer-Leoni}

Cette contribution sinscrit dans le champ de la didactique comparée et s'articule autour du concept de contrat didactique et de ses dimensions topogénetiques, chronogénetiques et mésogénetiques. Ces outils théoriques, habituellement mis en ouvre pour comprendre les pratiques scolaires, sont convoqués, à titre exploratoire, pour analyser des pratiques du contexte préscolaire. L'étude prend appui sur une tâche imaginée par un groupe d'enseignantes dans le but de la proposer à des élèves de quatre ans. La tâche est réalisée et étudiée dans deux contextes: en classe enfantine et dans un groupe d'enfants en crèche. Le but est la comparaison de ce que devient la tâche lorsqu'elle est précipitée dans deux institutions différentes mais qui, toutes les deux, prennent en charge des enfants de 4 ans. L'analyse se focalise sur une difficulté spécifique de la tâche identifiée à travers une analyse a priori de celle-ci. Les techniques des professionnelles (enseignante, versus éducatrice) pour gérer cette difficulté s'avèrent différentes même si leur finalité est fondamentalement la même.

\section{Une étude didactique dans le préscolaire}

Cet article explore ce que devient une tâche lorsqu'elle est gérée par des professionnel(le)s issu(e)s de formations différentes dans deux institutions distinctes. Létude fait partie d'un projet de plus vaste portée (Munch, thèse en cours) qui s'inscrit dans le champ de la didactique comparée (Mercier, Schubauer-Leoni \& Sensevy, 2002). La thèse consiste à étudier comment, dans le contexte des institutions genevoises prenant en charge des enfants de 4 ans, l'enfant est amené à passer d'une position de jeune enfant dans une Institution de la Petite Enfance (IPE) à celle d'écolier en institution préscolaire (1ère enfantine:1E). Afin de bien saisir les enjeux de ce passage, il faut garder à l'esprit que, dans le cas du canton de Genève, si l'entrée à l'école obligatoire est fixée à 5 ans et 10 mois au plus tôt (1ère primaire), deux années non obligatoires ${ }^{1}$ d'école enfantine $(1 \mathrm{E} ; 2 \mathrm{E})$ sont rattachées au contexte scolaire et sont confiées à des enseignant(e)s. A partir de 3 ans et 10 mois l'enfant peut donc entrer à l'école enfantine. Par ailleurs, dès l'âge 
de 4 mois et jusqu'à 4 ans et 9 mois, les enfants qui habitent ce canton peuvent fréquenter une IPE et sont alors pris en charge par des éducateurs(trices) du jeune enfant, dans des crèches, jardins d'enfants ou autres lieux d'accueil, sous le label IPE. A 4 ans, pour des raisons diverses que nous ne tenterons pas d'élucider ici, un enfant peut donc être déjà en $1 \mathrm{E}$ ou encore dans «un groupe de grands» en IPE.

Dans le cadre de cet article, nous limiterons notre propos à la discussion de la pertinence du système conceptuel adopté. Nous allons pour cela recourir à un ensemble très limité de données. Celles-ci seront utilisées en guise d'exemples à la fois de la méthode d'analyse et des critères d'interprétation des phénomènes. Conformément aux intentions du projet comparatiste en didactique, nous faisons le pari de la pertinence d'un cadrage conceptuel issu des théories didactiques (et en particulier de la didactique des mathématiques) pour comprendre non seulement les phénomènes scolaires mais aussi ceux qui caractérisent les apprentissages organisés dans le préscolaire et plus particulièrement en 1ère enfantine d'une part et en crèche d'autre part. Nous ne visons pourtant pas l'abaissement à des âges plus précoces des projets d'enseignement formels de la lecture, de l'écriture ou du calcul. Nous souhaitons plutôt attirer l'attention sur les objets qui deviennent enjeu de l'action conjointe d'enfants et d'éducateurs(trices) au sein de projets éducatifs en IPE versus l'action conjointe d'élèves et d'enseignant(e)s dès l'entrée à l'école enfantine (1E). Nous montrons ainsi la nécessité d'un modèle ternaire fortement déterminé non seulement par les modalités de fonctionnement des sujets en situation, mais aussi par la nature des tâches et des savoirs qu' elles comportent. L'insistance sur les objets s'accompagne de la prise en compte des institutions dans et par lesquelles les objets s'inscrivent dans des jeux d'attentes entre partenaires. En ce sens, nous nous donnons les moyens d'éprouver, dans deux institutions du préscolaire, la pertinence du recours à un cadre théorique, classiquement dévolu à l'analyse des situations d'enseignement à l'école.

\section{Le cadre conceptuel}

Quelques travaux existent déjà et analysent comment, par quels processus, l'enfant devient élève (Amigues \& Zerbato-Poudou, 2000; Garcion-Vautor, 2000) tout en montrant la pertinence de concepts didactiques pour éclairer le fonctionnement institutionnel de jeunes élèves aux prises avec les apprentissages premiers. Les travaux de Thévenaz-Christen (2002a et b) en didactique du français et ceux de Briand (1999) et de Pérez (1984) en didactique des mathématiques sont particulièrement éclairants à la fois pour saisir les enjeux éducatifs et didactiques qui animent les institutions et les modalités par lesquelles les sujets se situent dans des dynamiques d'apprentissages spécifiques, face aux attentes des professionnel(le)s en présence.

Pour notre part, l'inscription de la recherche dans le champ de la didactique comparée est un choix théorique fondamental. En effet, si cette approche vise la 
comparaison de phénomènes didactiques pour en dégager les spécificités et les généricités au gré des savoirs enseignés et appris dans les écoles, un projet de ce type peut d'autant mieux se développer s'il s'intéresse également à interroger "l'ailleurs du didactique " (au sens de Mercier, Schubauer-Leonie et Sensevy, 2002). Ce "détour» vise à éviter la réification, voire la naturalisation du scolaire en questionnant les critères (institutionnels et inhérents aux pratiques) de délimitation de la sphère que la culture courante considère comme étant le propre de l'école et donc le lieu par excellence des échanges d'enseignement et d'apprentissages. La didactique comparée étudie dès lors à la fois des lieux classiquement désignés comme ceux du didactique et d'autres lieux qui se revendiquent plutôt de l'éducatif. Ainsi, des institutions n'ayant pas officiellement un mandat d'enseignement, comme les crèches et jardins d'enfants, tout en étant chargées de la coéducation (avec les familles) du jeune enfant, s'avèrent particulièrement intéressantes pour travailler l'articulation entre le didactique et l'éducatif en problématisant ainsi la frontière trop vite tracée entre eux.

Dans ce champ théorique, il est désormais classique de considérer le concept de contrat didactique (Brousseau, 1990; Leutenegger \& Schubauer-Leoni, 2002; Sarrazy, 1995; Schubauer-Leoni, 1986) comme un concept majeur pour analyser un enseignement et son évolution négociée. Ce "contrat» ${ }^{2}$ fait exister trois instances intimement articulées: une instance élève, une instance enseignante et une instance constituée par les objets de savoir à enseigner et à apprendre. Il peut dès lors paraître surprenant d'importer le concept de contrat didactique (conçu pour gérer le rapport aux savoirs enseignés) dans des contextes qui disent n'avoir que des intentions éducatives (non spécifiques d'un savoir). Les conditions d'importation de ce concept nécessitent dès lors une vigilance épistémologique accrue en plaçant l'objet d'apprentissage au centre de cet examen et en posant explicitement la question des conditions qui sont créées pour modifier les connaissances des apprenants. Dans cette réflexion, les travaux de Chevallard (1992) sont éclairants. Son approche anthropologique du didactique a modélisé le lien entre attentes institutionnelles et modification des rapports aux objets dans les situations d'enseignement à l'école ainsi que dans des situations de la vie courante. En nous basant sur ce cadrage, nous avons déjà montré ce que permet le concept de contrat didactique pour analyser des enjeux d'apprentissage dans des crèches (Schubauer-Leoni, Munch \& Kunz-Félix 2002; Schubauer-Leoni \& Munch, 2003). Le rôle que vient jouer l'éducateur(trice) auprès de jeunes enfants peut, dans cette perspective, être assimilé à des gestes d'enseignement puisque des actions visant la modification du rapport à des objets peuvent être clairement identifiées en crèche aussi. Kunz-Félix, (1998) a notamment étudié l'évolution des rapports à l'objet «habillage après la sieste» en IPE. Dans notre modélisation, tout objet, pensé par les éducateurs(trices) comme enjeu des situations éducatives avec de jeunes enfants en IPE, vient ainsi occuper la place attribuée à des objets de savoir (inhérents, par exemple, à l'entrée dans la culture écrite) dans le contexte de l'école. 
Afin d'étudier l'évolution des systèmes observés, trois processus génétiques ont été mis en évidence (Chevallard, 1992; Sensevy, 2001; Sensevy, Mercier \& Schubauer-Leoni, 2000) et permettent d'explorer trois composantes majeures et fortement imbriquées du fonctionnement d'un contrat didactique et de l'action enseignante: la composante relative aux positionnements respectifs des partenaires du jeu éducatif (topogenèse); la composante temporelle (chronogenèse) et la composante inhérente aux objets à explorer et donc enjeu d'étude et d'apprentissage (mésogenèse). Plus précisément, nous dirons que: i/ La topogenèse renvoie aux positions (topos) occupées par les acteurs du système au fur et à mesure de son évolution (genèse du système). Dans le contexte scolaire, le système didactique est constitué d'une part de l'enseignant et d'autre part de l'élève ${ }^{3}$ chacun établissant des rapports distincts (pour l'un rapport d'enseignement, pour l'autre rapport d'apprentissage) aux objets. ii/ La chronogenèse rend compte des évolutions temporelles des objets qui sont enjeux du système observé, mais aussi de l'évolution des rapports de connaissance que les acteurs entretiennent avec ces objets. Cette genèse pose la question de l'articulation entre le tempo qu'impose l'enseignant à travers l'introduction incessante d'objets nouveaux avec le temps d'apprentissage du côté de l'élève. Le temps de l'apprenant et le temps didactique sont à situer dans les temporalités de l'institution et de ses horloges. Les unités temporelles pertinentes ne concernent pas uniquement des unités longues (mois, trimestre ou année), mais aussi des unités courtes à l'intérieur d'une leçon ou d'une activité. iii/ La mésogenèse renvoie, quant à elle, au système d'objets auquel l'apprenant est confronté pour modifier ses connaissances. Ce système d'objets, appelé milieu est l'élément médiateur (médius) de la relation didactique et éducative. A ce titre il est l'enjeu de négociations quant à sa fonction, son interprétation, le rapport qu'il convient d'entretenir à son égard à tel moment de l'activité. Nécessairement en transformation, le système d'objets auquel l'apprenant est confronté change quantitativement (des objets nouveaux sont proposés, d'autres disparaissent ou sont délaissés) et qualitativement (le rapport de connaissance en change le statut, les objets sont «reconstruits» sur d'autres plans: représentationnels, symboliques, mnémoniques, conceptuels). Pouvoir décrire l'émergence successive des objets introduits dans le milieu est donc un moyen privilégié pour saisir les enjeux du contrat didactique et les négociations qu'il permet.

Topogenèse, chronogenèse et mésogenèse, en tant que composantes dynamiques du contrat didactique, sont à la fois des critères d'organisation des données sur lesquelles s'appuie cette étude et des clefs pour décrire les réalités d'apprentissage, pour organiser l'analyse et charpenter les possibles interprétations des phénomènes. 


\section{La construction des observables et quelques repères méthodologiques en vue de l'analyse}

Les choix méthodologiques opérés pour interroger les deux institutions ont été décrits ailleurs (Schubauer-Leoni \& Leutenegger, 2002). Ce cadre méthodologique prend pour enjeu les systèmes ordinaires d'enseignement et d'éducation tout en optant pour une approche clinique de ces systèmes (Leutenegger, 2000) au sein de contraintes expérimentales précises (Schubauer-Leoni \& Leutenegger, 2002). Si cette approche situe l' observation de ce qui se passe en classe au centre des analyses, elle donne une place importante à l'analyse a priori des tâches observées. Ce type d'analyse devient dès lors un outil indispensable à la conception, à la gestion et à l'analyse de l'ensemble du dispositif et des données. Dans les travaux de l'équipe genevoise (notamment: Leutenegger, 2003; Leutenegger \& Schubauer-Leoni, 2002; Schubauer-Leoni \& Chiesa Millar, 2002) l'analyse clinique prend la forme d'un jeu articulé de divers corpus afin de réduire les incertitudes d'interprétation: ces corpus renvoient aux productions des élèves, aux entretiens avec les enseignants sur leur projet spécifique d'enseignement et aux entretiens, conduits après le travail en classe, avec les élèves.

Dans le cas de cette étude, afin d'interroger les pratiques d'éducateurs(trices) et d'enseignant(e)s, nous avons choisi de focaliser l'observation sur une même tâche ${ }^{4}$. La tâche dont il va être question est le résultat d'une démarche ad hoc au service des dimensions à la fois cliniques et expérimentales du dispositif. Sa particularité est d'avoir été imaginée par trois enseignantes à qui la recherche a confié le rôle collectif de conceptrices. Nous leur avons demandé d'inventer une tâche/activité typique de ce qui leur paraît ordinairement réalisable avec des élèves fréquentant une classe de $1 \mathrm{E}$ en milieu d'année ${ }^{5}$. C'est donc cette tâche, nommée par les enseignantes/conceptrices "Activité 1E» (annexe 1), qui va être le pivot des observations traitées dans cet article. Nous allons donc présenter quelques éléments majeurs de son analyse a priori. Nous nous focaliserons ensuite sur sa réalisation: d'une part dans un contexte préscolaire auprès d'une enseignante et de sa classe et d'autre part auprès d'une éducatrice et de son groupe d'enfants en crèche. Nos analyses seront éclairées, chaque fois que nécessaire, par le discours recueilli auprès des professionnelles, lors d'entretiens à propos de cette tâche. Il s'agit donc de montrer, dans l'espace réservé à cet article, les niveaux d'analyse que comporte l'approche clinique et expérimentale qui est la nôtre. Nous soulignons donc la vocation à la fois théorique et méthodologique de la présente contribution.

\section{Quelques phénomènes et leur interprétation}

\section{L'analyse a priori de la tâche}

Pour pouvoir comprendre les enjeux de la tâche, les opérations de pensée qu'elle 
comporte et les interprétations qu'elle peut véhiculer à la fois du côté de l'enseignante et de l'éducatrice, mais aussi des élèves/versus enfants, il convient de procéder à une analyse fine de ses composantes. En effet, nous admettons avec Clot (1998), qu'il est utile de distinguer tâche et activité afin de saisir ce que devient la tâche lorsqu'elle est précipitée dans le vif des interactions didactiques et éducatives en se faisant ainsi activité (Schubauer-Leoni, 2002). La mise en évidence des contraintes et des possibles de la tâche susceptibles de structurer l'activité, paraît donc une étape indispensable de l'analyse sans toutefois épuiser les enjeux de l'activité. Celle-ci fera l'objet d'un niveau d'analyse ultérieur.

L'analyse a priori de la tâche "Activité 1E» (Munch, 2002) a notamment mis en évidence le rôle que vient jouer le matériel (quantité et caractéristiques), le nombre d'enfants concernés et le fait que les enfants peuvent à la fois tenir un rôle de meneur et de duplicateur (position qui met en jeu des savoirs distincts). Si l'objectif majeur visé par la tâche est un enjeu cognitif qui s'articule autour de la notion de position relative des objets selon qu'ils se trouvent respectivement dessus, dessous, à côté, à gauche ou à droite, le déroulement, tel qu'il est formulé dans la version écrite de la tâche (version "prescrite» au sens des ergonomes), pose, quant à lui, un certain nombre de problèmes pour une possible gestion de l'activité. En effet, la tâche prévoit le déroulement suivant: «Le meneur construit son château en décrivant ce qu'il fait; les autres le font en même temps». Ceci suppose une gestion de la simultanéité entre le faire (gestes de pose des pièces constitutives du château) du côté du meneur, la description de l'action (gestes de formulation de l'action accomplie ou en train d'être accomplie) également du côté du meneur et l'enregistrement des informations ainsi que leur traduction subséquente en gestes d'action du côté du duplicateur. Ce découpage de la procédure de réalisation montre que l'unité qui sera décrite par le meneur sera décisive pour permettre le travail de construction du duplicateur. Or, l'identification, voire le choix de l'unité à énoncer est une opération cognitivement complexe que le sujet n'anticipe pas nécessairement et qu'il découvre «en faisant», sans reconduire la même unité de façon stable ni tirer parti des effets du côté du duplicateur. L'analyse a priori a également mis en évidence que pour que l'activité se déroule selon des conditions aptes à permettre la réalisation de constructions identiques, cela demande un bagage langagier, de nomination des objets physiques et des actions, articulé aux compétences complexes au niveau de la spatialisation (recours à des référents externes ou à la notion de gauche/droite). Nous en concluons que, du côté d'enfants âgés de 4 ans, des stratégies expertes s'avèrent très peu probables sans le guidage de l'adulte.

Compte tenu de ce que révèle l'analyse a priori de la tâche, dans un premier temps, nous interrogerons les modalités de gestion globale que se donne l'éducatrice, respectivement l'enseignante, en cours d'activité. La consigne qui leur est donnée, via le descriptif de la tâche, laisse effectivement une large part d'interprétation au rôle de l'action des professionnelles. Dans un deuxième temps, nous nous focaliserons sur la question de l'unité énoncée par le meneur, puisque nous 
avons vu qu'elle comporte des difficultés importantes pour des enfants de cet âge. Nous questionnerons en conséquence l'action qu'engagent les deux professionnelles pour «tenir» l'activité nonobstant ces difficultés.

\section{Deux contextes de réalisation de la tâche: l'activité gérée par une enseignante (1E) et par une éducatrice (crèche)}

A la fois l'éducatrice et l'enseignante observées ont une expérience préalable de la prise en charge de ce groupe d'âge. L'éducatrice travaille dans le cadre d'une crèche subventionnée par la Ville de Genève, l'enseignante travaille dans une école publique en campagne genevoise. Aucune des deux n'a participé à la conception de la tâche, elles la découvrent donc à cette occasion. Dans ce sens, la comparaison devrait donner des indications sur la manière dont va vivre cette tâche à l'intérieur des habitudes que les deux professionnelles entretiennent avec les enfants/élèves dont elles s'occupent. Comment vont-elles accueillir la tâche? Quelles conditions vont-elles mettre en place pour la réaliser?

Une incursion du côté de l'entretien (préalable) avec l'enseignante, nous confirme qu'elle reconnaît la pertinence de la tâche («c'est tout à fait une activité de première enfantine»). L'éducatrice en revanche répond «non» lorsqu'on lui demande si elle a déjà fait des choses qui ressemblent, mais ajoute: «j’aimerais vraiment voir l'activité faite avec les enfants». Dans les deux cas, la tâche paraît faisable, quoique davantage «hors contrat» du côté de l'éducatrice. Celle-ci choisit de modifier la quantité de plots: «j'en mettrais quatre avec un de chaque couleur primaire [...] carré». Elle estime que la tâche va permettre de «mettre dessus, devant, derrière, nommer, apprendre à écouter un camarade, comparer semblable/différent». L'enseignante, tout en affirmant d'abord que "c'est égal», dira qu'elle va utiliser «les plots classiques [...] quatre couleurs [...] huit plots rectangulaires».

L'analyse a priori avait mis en évidence la fonction du matériel (nombre et type de plots, couleurs) dans la mobilisation des savoirs en jeu. Cependant, force est d'admettre que les professionnelles n'ont pas l'habitude d'engager des analyses a priori des possibilités cognitives et sociales que comporte la tâche, et dans le cas présent, elles ne semblent pas percevoir le rôle que vient jouer, précisément, le choix des pièces constitutives de la construction et l'organisation sociale de l'activité, ce qui les amène à apprécier globalement la tâche et sa faisabilité avec leurs élèves/enfants.

\section{L'analyse globale des deux séances: l'émergence de deux activités?}

Nous proposons une première présentation des observations par le biais de deux tableaux qui constituent deux réductions du déroulement des activités dans les deux contextes. Ces réductions sont faites sur la base de l'enregistrement vidéo et permettent d'identifier la succession des phases de construction et d'échange sur ces constructions. Volontairement très épurés, ces tableaux permettent de ne pas perdre de vue la globalité des deux séances. 
Tableau 1: La tâche dans les deux contextes

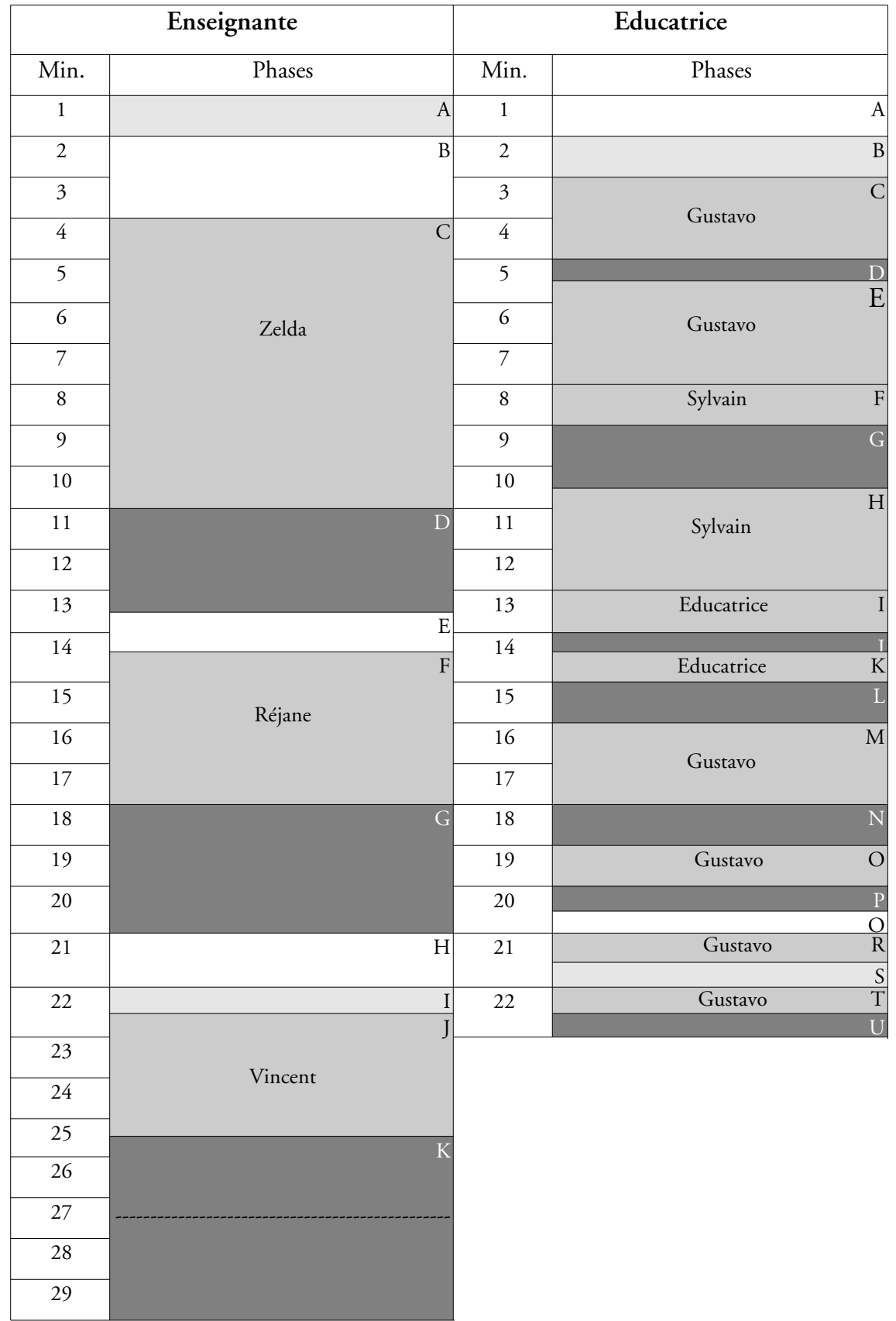


Les constats comparatifs suivants sont possibles:

- L'activité gérée par l'enseignante dure vingt-neuf minutes. Trois élèves sont meneurs: Zelda, Réjane et Vincent. La première construction faite par Zelda (C) dure sept minutes, la deuxième, réalisée par Réjane $(\mathrm{F})$ trois minutes et 30 secondes et la troisième de Vincent dure trois minutes $(\mathrm{J})$. Trois phases de bilan $^{6}$ ont lieu et suivent chaque phase de construction (D; G; K) ce qui représente en tout dix minutes de bilan. L'activité commence par la verbalisation de la consigne par l'enseignante (A, 1 minute), puis, deux minutes sont nécessaires pour installer les élèves et vérifier le matériel $(B)$. D'autres moments d'organisation sont observables (phase E) et plus spécifiquement une phase $(\mathrm{H})$ durant laquelle deux participants sont remplacés, ce qui entraîne une nouvelle verbalisation de consigne (I). Relevons encore que la cloche de la récréation sonne après vingt-six minutes et trente secondes. Il est donc probable que l'activité ait été prévue par l'enseignante comme devant durer environ vingt-cinq minutes.

- L'activité gérée par l'éducatrice dure vingt-deux minutes. Deux enfants sont meneurs: Gustavo et Sylvain. Gustavo occupe, pendant neuf minutes, six phases de construction (C; E; M; O; R; T). Sylvain, quant à lui, réalise deux constructions $(\mathrm{F} ; \mathrm{H})$ qui durent trois minutes en tout. L'éducatrice assume, à deux reprises, le rôle de meneur et réalise deux constructions (I; K) (1,30 min. en tout). Sept phases de bilan ont lieu (D; G; J; L; N; P; U) qui suivent certaines constructions. Certains moments (phases: E; H; R) de construction ne sont pas suivis de bilan. Dans deux cas, c'est une nouvelle construction qui s'enchaîne, alors que dans un cas c'est une nouvelle verbalisation de consigne (S). Relevons encore qu'un participant est remplacé (Q). L'activité a commencé, dans ce cas, par une distribution de matériel (A, 1 min.), suivie de la verbalisation de la consigne par l'éducatrice (B) qui a également duré une minute.

La comparaison de ces deux tableaux de réduction de l'information montre une dynamique générale qui differe sur certains points. Le milieu créé par chaque professionnelle et auquel l'élève/enfant est confronté est, en première approximation, différent; par conséquent, la mésogenèse, en tant que construction progressive d'un milieu supposé partagé entre les deux professionnelles et les apprenants diverge sur certains aspects tout en comportant des points de concordance. Nous y reviendrons. Commençons par relever que l'activité organisée par l'enseignante dure sept minutes de plus, alors que le nombre de constructions est inférieur dans son cas (dix constructions en IPE, trois en 1E). Le matériel en jeu peut éclairer cette différence, puisque faire une construction avec quatre cubes prend moins de temps que de faire une construction avec sept parallélépipèdes (et non huit comme initialement prévu par l'enseignante). En comparant les autres phases, nous constatons que l'éducatrice commence par distribuer le matériel et procède à la formulation de la consigne après, alors que la consigne vient 
dans un premier temps pour l'enseignante. Dans les deux cas, cette formulation prend place dans les deux premières minutes de l'observation. C'est donc très rapidement que les enfants/élèves sont placés face au problème. Les topos distincts sont visibles: dans cette phase, les professionnelles organisent le milieu, les élèves attendent de connaître leur rôle. Contrairement à l'éducatrice, l'enseignante procède à une vérification du matériel comme suggéré par les concepteurs. Par la suite, et dans les deux contextes, des phases de construction s'enchaînent avec des phases de bilan. Nous constatons alors que le nombre de bilans est supérieur du côté de l'éducatrice, mais qu'en terme de durée, ils sont plus longs du côté de l'enseignante. Dans les deux cas, autour de la vingt-deuxième minute, les professionnelles procèdent à des changements dans le groupe d'enfants/élèves: un participant est remplacé dans le groupe d'enfants alors que deux le sont dans le groupe d'élèves. A cette occasion et dans les deux cas, nous assistons à une reprise de la consigne. Soulignons encore, et la différence est de taille, que l'éducatrice va occuper le rôle de meneur, alors que l'enseignante garde sa position de gestionnaire de l'ensemble de l'activité à l'extérieur des positions de meneur et de duplicateur.

\section{L'analyse de phases de construction en lien avec la problématique de l'unité d'action et de description/formulation}

L'analyse va se focaliser maintenant sur le révélateur majeur de la difficulté de la tâche: le choix de l'unité d'action et de description/formulation. Il s'agit, nous l'avons vu, de gérer la simultanéité prévue dans le descriptif de la tâche prescrite: «Le meneur construit son château en décrivant ce qu'il fait; les autres le font en même temps".

A partir de la transcription sous forme de protocole des séances, nous savons que l'enseignante, à la cinquième minute, dit à Zelda (élève qui occupe en premier le rôle de meneur): «je vais te donner l'indication / chaque fois que tu vas mettre quelque chose pour construire ton château» (Zelda avait annoncé son intention de construire un "château »). L'enseignante intervient donc directement dans la structuration de l'action du meneur et ce faisant elle participe à la réalisation de la tâche. C'est elle qui va prendre en charge la gestion des unités d'action et de description/formulation, ce qui revient à scander le déroulement de l'activité.

Du côté de l'éducatrice, l'analyse du protocole de la séance ne nous montre pas d'indice verbal de cet ordre. Pourtant, Gustavo rencontre la même difficulté que Zelda au début de l'activité et devra, comme Zelda, parvenir à décrire les unités. Nous proposons donc d'analyser plus en détail comment l'éducatrice gère cette difficulté. Le tableau 2 reprend les phases du tableau 1 en ajoutant les appellations par lesquelles les enfants nomment leurs différentes constructions («un carré», un "crochet», etc.) ainsi que les interventions verbales de l'éducatrice qui rythment l'avancement de l'activité. La colonne de droite donne la représentation schématisée des dix constructions ${ }^{7}$ qui sont réalisées par les meneurs durant cette activité. 
Tableau 2: Dix phases de construction en crèche

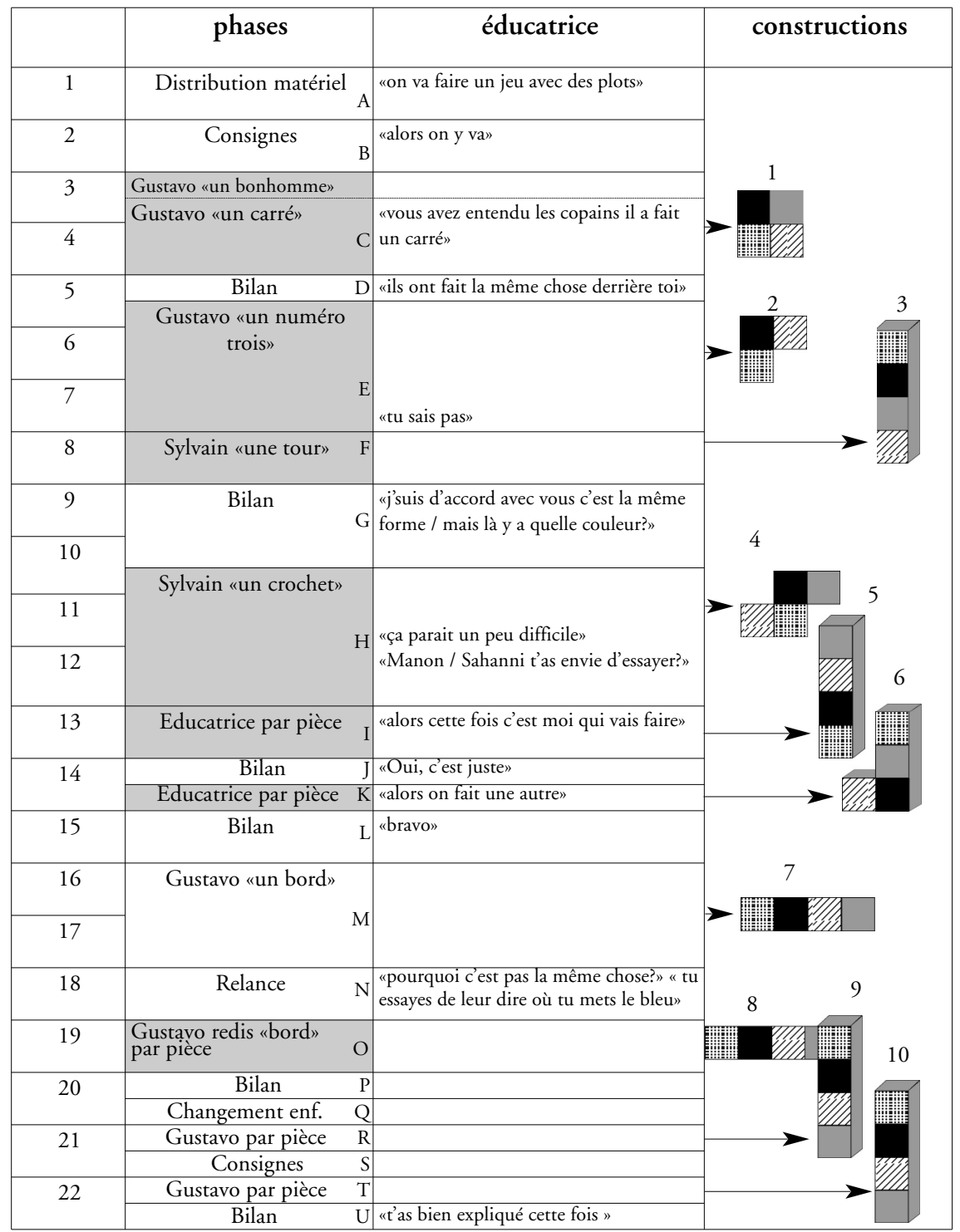

Gustavo va proposer diverses nominations pour ses constructions («un bonhomme», "un carré», "un numéro trois», «un bord») puis réalisera une construction décrite pièce par pièce («on met d'abord le jaune / après le rouge dessus le jaune et après le bleu dessus le rouge et le vert dessus le bleu»). Ceci revient à une 
gestion articulée des unités d'action et de description/formulation. Sylvain, quant à lui, propose «une tour» puis «un crochet». Le tableau 1 nous avait permis de constater que seules certaines constructions étaient suivies par un bilan. Nous allons ici brièvement évoquer le contenu de ces bilans et en clarifier ainsi le statut. Le «bonhomme» proposé par Gustavo permettra à l'éducatrice de redire la consigne et de donner l'indice de l'importance des couleurs, ce qui ne sera pourtant pas repris par Gustavo à cette étape du déroulement. Pour répondre à la question de l'éducatrice qui lui demande: «qu'est-ce que tu fais là», Gustavo répond: «un carré». Les trois duplicateurs vont effectivement réaliser un "carré». Et l'éducatrice commentera: «ils ont fait la même chose derrière toi». Or, si la forme finale du château est bien la même, l'organisation des couleurs n'est pas respectée, il ne s'agit donc pas de constructions identiques. L'éducatrice renonce, ici, à une contrainte de la tâche (les constructions sont censées être identiques sur tous les plans) au profit d'une valorisation d'une composante de la production réalisée. Ce type de comportement, qui pourrait faire penser à une négociation «à la baisse» du contrat didactique, est très répandu dans les pratiques enseignantes à tous les niveaux de la scolarité et montre très clairement le souci, tout à fait justifié, des professionnel(le)s de ne pas décourager les élèves au moment même où ils investissent la tâche. L'adulte n'est certainement pas dupe, il agit donc ainsi afin de tenir l'apprenant dans une tension utile à la modification du rapport au savoir tout en préservant le cadre de la relation didactique et éducative.

Dans le cas de la situation de crèche observée, la proposition de Gustavo de faire «un numéro trois» ne permet pas aux duplicateurs de faire une construction. L'éducatrice n'obtient pas de Gustavo qu'il énonce les unités de description et elle conclut: "tu sais pas», sous-entendant "tu ne sais pas comment décrire les unités permettant aux duplicateurs de refaire la construction que tu nommes un numéro trois». A cette étape, aucun bilan n'est réalisé avec les participants. Sylvain, sollicité par l'éducatrice pour être meneur, propose «une tour». Comme dans le cas du "carré», les duplicateurs parviennent à faire une construction identique au niveau de la forme, mais pas au niveau de l'organisation des couleurs. Cependant, contrairement à son intervention concernant le "carré», l'éducatrice n'accepte pas de dire que c'est la même chose et déclarera durant le bilan: «j'suis d'accord avec vous c'est la même forme / mais là y a quelle couleur?». Les enfants, invités à s'intéresser aux couleurs, remarquent que celles-ci ne sont pas placées au même endroit. L'éducatrice modifie ainsi les attentes à l'égard des enfants en manifestant une exigence supplémentaire: l'emplacement des cubes en fonction de la couleur. Sylvain est alors appelé par l'éducatrice à refaire: espère-t-elle que Sylvain puisse prendre en compte les éléments du bilan et qu'il refera ainsi une tour en disant aussi les couleurs? Mais Sylvain propose cette fois "un crochet». Comme dans le cas du «numéro trois» de Gustavo, les duplicateurs ne construisent rien. Il convient dès lors de noter que l'évocation de certaines constructions comme "carré» et «tour» renvoie à des modèles culturels suffisamment disponibles chez les enfants pour leur permettre la reproduction de la bonne forme (en 
utilisant tous les plots à leur disposition). D'autres constructions plus originales («un crochet», "un numéros trois» ou "un bord») s'avèrent plus difficilement reproductibles sans une procédure complexe d'enchaînement d'actions successives (séquentialisation). Aucun bilan commun s'ensuit, sinon sous la forme d'un commentaire de l'éducatrice: "ça paraît un peu difficile». L'éducatrice demande alors à Manon et Sahanni si elles souhaitent occuper le rôle de meneur. Les deux filles refusent. La situation semble dès lors bloquée puisque, jusque là, aucun enfant ne recourt au découpage de l'action sous la forme de description/formulation en unités de couleur.

C'est à ce moment (min 13) que l'éducatrice va occuper le rôle de meneur en proposant, elle aussi, de faire une tour. Elle opte ainsi pour une modification minime du milieu: la tour a déjà été réalisée avec succès, en tant qu'empilement des pièces, mais sans respecter les couleurs, c'est à cette composante du milieu qu' elle s'attache puisqu' elle procède en disant la couleur du cube pris et, en scandant ses gestes, elle demande "vous l'avez dans la main?», "vous avez fait?», "vous avez tous fait?». Elle montre ce faisant un type de geste nouveau: pour permettre à l'autre de dupliquer il faut lui faire une place temporelle d'action. Ceci permet la correspondance terme à terme entre la pièce posée par le constructeur et la pièce posée par le duplicateur. Les constructions des duplicateurs seront identiques et l'éducatrice conclut, lors du bilan (min 14), "oui c'est juste». Les enfants semblent avoir compris et Sylvain dira même, depuis la position d'observateur qu'il occupe (le matériel est prévu pour quatre participants): «c'est trop facile». L'éducatrice reprend le rôle de meneur et réalise la sixième construction. Les duplicateurs feront tous la même construction qu'elle. Sylvain, qui a toujours le rôle d'observateur, dira à nouveau, lors du moment de discussion qui suit: «tu leur dis quand tu prends / c'est trop facile comme ça». Ce commentaire met en évidence une interprétation de la tâche du meneur qui avait été sous-estimée: le constructeur/meneur pense devoir faire en sorte que les duplicateurs «aient de la difficulté» à faire leur construction. Dans ce cas, l'enjeu d'une réussite de tout le groupe ne semble manifestement pas partagé!

Ce sera Gustavo, autoproclamé meneur, qui va faire la septième construction. Il va proposer «un bord» que les duplicateurs ne parviendront pas à refaire. A cette étape, l'éducatrice laisse les duplicateurs face à cette impossibilité et la discute. Cependant, le contenu de la discussion s'adresse principalement à Gustavo et prend dès lors la forme d'une relance sous forme d'aparté: «comment veux-tu qu'ils sachent [...] tu leur as pas dit», et finira par affirmer "tu essayes de leur dire où tu mets le bleu» et Gustavo dira «j'ai mis le bleu à côté du rouge et le rouge et aussi le bleu à côté du vert et le rouge à côté du jaune». A la fin de sa description/formulation, la discussion s'amorce et l'éducatrice observe que Sylvain «a la même chose». A la suite du changement de participant, Gustavo va encore réaliser deux constructions en hauteur qu'il décrira/formulera par unité (ce qui semble attester une modification de son rapport à l'objet). Pourtant, les duplicateurs ne parviennent pas à suivre ce qui est dit et les constructions ne sont pas les 
mêmes. Face au constat de Gustavo: «Dan il a pas la même chose» (sous-entendant probablement: "alors que j'ai décrit une pièce après l'autre»), l'éducatrice conclura l'activité en disant: «ben non parce qu'ils ont pas écouté /// pourtant t'as bien expliqué cette fois /// voilà».

\section{La co-évolution mésogénétique, topogénétique et chronogénétique}

L'évolution des objets a mis en évidence leur nature au fur et à mesure de l'avancement de la séance: les objets se manifestent d'abord sous la forme de plots physiques à manier et à agencer, ils s'avèrent ensuite positionnés les uns par rapport aux autres (objet cognitif complexe à élaborer conceptuellement en fonction des positions relatives). Au cours de la séance, c'est la nomination de l'objet construit (un «carré», une «tour», ...) qui a d'abord pris le dessus. Or, cette désignation de fin de travail du meneur nécessite de la part de l'éducatrice une "déconstruction" de cet objet matériel et langagier au profit de l'objet «unité de description/formulation pour chaque geste de construction» de la part du meneur. Mais, nous l'avons vu, cet objet d'apprentissage est, comme prévu dans l'analyse a priori, doublement résistant pour le meneur: cognitivement (il est difficile d'isoler l'unité d'action et d'anticiper sur le temps de duplication du côté des camarades) et socialement (le meneur -c'est le cas de Sylvain- peut avoir envie d'empêcher la bonne construction en pensant ainsi valoriser son propre rôle). La mésogenèse apparaît dès lors laborieuse et oblige l'éducatrice à venir occuper le rôle de meneur afin de montrer l'utilité des unités de description/formulation. Du point de vue topogénétique, les systèmes de places attribués aux enfants, selon qu'ils sont en position de meneur, de duplicateur ou encore, d'observateur (le cas de Sylvain lorsque l'éducatrice joue le rôle de meneur) et celui occupé par l'éducatrice (tour à tour régulatrice de l'organisation successive de l'activité, meneur, et acteur habilité à dire la conformité/non conformité des rapports aux objets du moment) sont clairement visibles et intimement liés à la mésogenèse. De même, une lecture chronogénétique de la séance montre comment l'éducatrice crée une temporalité par la désignation successive d'objets nouveaux au fil de l'activité. Depuis leurs topos respectifs, les enfants s'inscrivent dans cette temporalité en interprétant, à leur façon, les contraintes et en «bloquant» l'avancement par l'introduction de l'objet «nomination de la construction finie». La «monstration» par l'éducatrice permet dès lors à Gustavo d'entrer dans la logique "travail par unité ", mais il faut bien remarquer qu'il s'agit de l'enfant qui a eu le plus d'occasions d'exercer le rôle de meneur. Grâce à la performance de cet enfant, l'éducatrice peut clore la séance avec un bilan prometteur: "t'as bien expliqué cette fois» en montrant par-là l'aboutissement d'un rapport adéquat aux objets de la séance.

Au niveau topogénétique, si nous comparons la séance de l'éducatrice avec celle conduite par l'enseignante, nous pouvons penser que si l'enseignante ne prend pas la position de meneur c'est notamment parce qu'elle a pu obtenir d'un élève qu'il coordonne ses gestes et ses paroles afin de permettre aux duplicateurs de venir occuper leur position dans des conditions acceptables, voire efficaces. Du 
point de vue chronogénétique, nous relèverons aussi qu'à l'intérieur du temps institutionnel que se sont octroyées l'éducatrice et l'enseignante, elles visent un déplacement des enjeux de l'activité vers l'usage d'unités d'action et de description/formulation. Elles parviennent d'ailleurs à obtenir des constructions idoines. Leur technique differe, mais le résultat est du même ordre. En quelque sorte, on peut dire que le milieu visé (et en particulier la nécessité de traiter l'objet unité de description/formulation) est le même dans les deux cas, la façon de le faire vivre oblige pourtant les deux professionnelles à faire des choix topogénétiques distincts: l'une jouant le rôle de meneur faute de trouver le moyen de le faire jouer correctement par un enfant, l'autre trouvant parmi ses élèves un sujet pouvant assumer ce rôle.

\section{En guise de conclusion}

Les éléments d'analyse que nous avons mis en évidence renvoient aux enjeux du contrat didactique et éducatif dans les deux contextes institutionnels. En effet, dans les deux cas, des règles implicites permettent à l'activité de se dérouler tout en créant des formes évolutives d'intersubjectivité à propos des constructions et reconstructions. L'enseignante et l'éducatrice jouent notamment toutes les deux le rôle de régulatrices de l'activité, distribuant des rôles spécifiques et conjoncturels aux enfants/élèves qui se voient d'ailleurs attribuer des gestes ciblés et ordonnés à tenir (phénomènes topogénétiques constitutifs du contrat). Cependant, dans le cas de l'éducatrice, les deux enfants qui ont été sollicitées pour «essayer» vont décliner l'offre. A noter que des comportements de ce type auraient pu se manifester aussi en $1 \mathrm{E}$, les élèves étant à peine initiés aux enjeux de ce type d'interpellation. Le sentiment d' "obligation» à faire ce qui est demandé et qui comporte une forme d'assujettissement aux attentes institutionnelles, serait-il le propre de l'école «obligatoire» dont l'école enfantine serait le sas d'entrée?

Que ce soit en IPE ou à l'Ecole, les jeunes enfants ou écoliers de 4 ans que nous avons considérés ici se sont tous heurtés aux difficultés considérables de cette tâche et dans les deux cas, les professionnelles ont indiqué la technique consistant à décomposer l'action en unités de gestes. En jouant le rôle de chef d'orchestre, elles ont rythmé l'unité de mouvement (geste et parole) du meneur pour qu'il résonne (et raisonne) du côté des duplicateurs. Les deux professionnelles ne se distinguent pas fondamentalement sur cette tâche et nous allons jusqu'à dire que si l'analyse avait été faite à l'aveugle, sans savoir dans quel cas la séance était animée par une éducatrice ou par une enseignante, on aurait eu bien de la peine à argumenter en faveur d'une attribution à l'une ou l'autre professionnelle. Ce résultat, bien que limité à ces deux observations, est de taille dans la mesure où il nous oblige à considérer que, toutes choses différentes par ailleurs (Institutions et donc projets éducatifs distincts, formation différente des professionnelles, personnes qui ne se connaissent pas), la tâche engendre des actions d'en- 
seignement du même ordre. Dans une perspective de description et de compréhension de la dynamique des deux séances, les dimensions méso-, topo- et chronogénétiques s'avèrent des descripteurs efficaces pour mener DES analyses dans les deux contextes de préscolarité. Cette étude ne permet, certes, aucune généralisation. Seule l'analyse du devenir de la tâche prescrite chez d'autres professionnel(le)s - éducateurs(trices) et enseignant(e)s - permettra de tirer quelques conséquences quant à la spécificité et généricité des pratiques engendrées par cette tâche, à la fois du côté des professionnel(le)s et des apprenants dans deux institutions à première vue aussi diverses quant à leurs finalités.

\section{Notes}

1 D'après 1,Observatoire de la petite enfance (2001), en 2000, parmi les enfants résidant dans le canton de Genève, $79 \%$ des enfants âgés de 4 ans et la quasi totalité de ceux de 5 ans sont scolarisés.

2 Les guillemets s,imposent puisque il s,agit $d$, un contrat sui generis, fait en partie de règles stables (quoique la plupart du temps implicites), mais surtout de règles en constante mutation qui permettent la progression des enjeux de connaissance. A juste titre, G. Brousseau (1990) caractérise la négociation de ce contrat comme relevant d,une recherche constante d,un contrat entre partenaires.

3 Le topos d'élève est, en fait, occupé par plusieurs positions en fonction notamment du degré d'adéquation des sujets aux attentes des enseignants.

4 La tâche constitue la variable contrôlée du dispositif expérimental.

5 Le dispositif général de la recherche (Munch, thèse en cours) prévoit la même demande auprès d'un groupe de trois éducatrices devant inventer une tâche/activité typique de ce qui leur paraît ordinairement réalisable avec des enfants fréquentant un groupe de 3-4 ans en IPE en milieu d'année. Nous ne traiterons pas de ces données ici.

6 Nous désignons par «bilan» les phases dans lesquelles l'adulte donne des indices d'acceptation ou de non acceptation de la construction réalisée. Ces phases assurent une forme d'institutionnalisation de ce qu'il convient de faire ou dire à tel moment de la séance. Ces phases apparaissent en grisé foncé dans le tableau, alors que les phases de consigne sont en grisé clair. Un grisé intermédiaire est utilisé pour les phases de construction (avec le nom des meneurs). En blanc les phases de transition sans bilan.

7 Les représentations en deux dimensions se réferent aux constructions «à plat», alors que les représentations en volume évoquent une construction en hauteur. Du point de vue des couleurs: cube jaune $=$ gris, cube rouge $=$ rayé, cube vert $=$ quadrillé, cube bleu $=$ noir .

\section{Références bibliographiques}

Amigues, R. \& Zerbato-Poudou, M.-T. (2000). Comment l'enfant devient élève. Paris: Retz.

Briand, J. (1999). Contribution à la réorganisation des savoirs pré-numériques et numériques, études et réalisation d'une situation d'enseignement de l'énumération dans le domaine pré-numérique. Recherches en Didactique des Mathématiques, 19 (1), 41-76.

Brousseau, G. (1990). Le contrat didactique: le milieu. Recherches en Didactique des mathématiques, 9 (3), 309-336.

Clot, Y. (1998). Le travail sans l'homme? Pour une psychologie des milieux de travail et de vie. Paris: La Dispute (2ème éd.). 
Chevallard, Y. (1985). La transposition didactique. Du savoir savant au savoir enseigné. Grenoble: La Pensée Sauvage (2ème éd. augm.).

Chevallard, Y. (1992). Concepts fondamentaux de la didactique: perspectives apportées par une approche anthropologique. Recherches en Didactique des Mathématiques, 12 (1), 73 112.

Kunz-Felix, M. (1998). L'éducation du jeune enfant à la lumière des théories didactiques: les enjeux de la relation éducateur du jeune enfant - enfant - objet d'apprentissage dans une démarche réflexive sur l'action éducative . Mémoire de licence, Université de Genève, Faculté de Psychologie et des Sciences de l'éducation, Genève.

Garcion-Vautor, L. (2000). L'entrée dans le contrat didactique à l'école maternelle: le rôle des rituels dans la construction d'un milieu pour apprendre. Thèse de doctorat de l'université AixMarseille 1.

Leutenegger, F. (2000). Construction d'une clinique pour le didactique. Une étude des phénomènes temporels de l'enseignement. Recherches en Didactique des Mathématiques, 20 (2), 209-250.

Leutenegger, F. (2003). Etude des interactions didactiques en classe de mathématiques: un prototype méthodologique. Bulletin de Psychologie, 466, 56(4), 559-571.

Leutenegger, F. \& Schubauer-Leoni, M.L. (2002). Les élèves et leur rapport au contrat didactique: une perspective de didactique comparée. In A. Terrisse (Ed.), Les didactiques scientifiques et technologiques. (Les dossiers des sciences de l'éducation $\mathrm{N}^{\circ} 8$, pp. 73-86). Toulouse: Presses Universitaires du Mirail.

Mercier, A., Schubauer-Leoni, M.L. \& Sensevy, G. (Ed). (2002). Vers une didactique comparée. Revue Française de Pédagogie, 141, 5-16.

Munch, A.-M. (2002). L'analyse a priori de deux tâches comme révélateur des attentes de deux institutions: l'Institution de la petite enfance et l'Ecole enfantine à Genève, Actes du 8ème congrès international de la DFLM, (Neuchâtel, 26-28 septembre 2001). [CD-ROM]. Neuchâtel: DFLM, INRP.

Munch, A.-M. (thèse en cours). L'éducation/enseignement préscolaire à la lumière de la didactique comparée. L'étude de deux tâches/activités comme éléments des attentes en Institution de la petite enfance et à l'école enfantine à Genève, Université de Genève, Faculté de psychologie et des sciences de l'éducation, Genève.

Observatoire De La Petite Enfance (2001). La petite enfance en Ville de Genève, indicateurs et tendances, 2001/3. Genève: Délégation pour la petite enfance.

Perez, J. (1984). Utilisation d'une théorie des situations en vue de l'identification des phénomènes didactiques au cours d'apprentissages scolaires: construction d'un code de désignation d'objets à l'école maternelle. Thèse de l'Université de Bordeaux II.

Sarrazy, B. (1995). Le contrat didactique. Note de synthèse. Revue Française de pédagogie, 85-118

Schubauer-Leoni, M.L. (1986). Le contrat didactique: un cadre interprétatif pour comprendre les savoirs manifestés par les élèves en mathématiques. Journal européen de psychologie de l'éducation, 2, 139-153

Schubauer-Leoni, M.L. (2002). L'analyse de la tâche dans une approche de didactique comparée. Actes du 8ème congrès international de la DFLM, (Neuchâtel, 26-28 septembre 2001). [CD-ROM]. Neuchâtel: DFLM, INRP

Schubauer-Leoni, M.L. \& Munch, A.-M. (2003). Un jeu de société dans une institution de la petite enfance: analyse didactique des interactions entre une éducatrices et cinq enfants à propos d'un jeu. Bulletin de Psychologie, 466, 56(4), 559-571.

Schubauer-Leoni, M.L., Munch, A.-M. \& Kunz-Felix, M. (2002). Comprendre les pratiques professionnelles dans les institutions de la petite enfance: intérêt de l'approche didactique. In M.-P. Trinquier, M.-T. Zerbato-Poudou (Ed.), Le pré-scolaire en questions: questions sur les pratiques. (Les dossiers des Sciences de l'éducation, 7, pp. 21-31). Toulouse: Presse Universitaire du Mirail. 
Schubauer-Leoni, M.L. \& Leutenegger, F. (2002). Expliquer et comprendre dans une approche clinique/expérimentale du didactique «ordinaire». In F. Leutenegger \& M. Saada Robert (Ed.), Expliquer et comprendre en sciences de l'éducation (pp. 227-251). Paris, Bruxelles: De Boeck Université, Collection Raisons Educatives.

Schubauer-Leoni, M.L. \& Chiesa Millar, V. (2002). Une «tâche de français sur un thème de géographie»: actions didactiques de l'enseignante dans le vif de l'activité en classe et dans son discours a priori. Revue Française de Pédagogie, 141, 123-134.

Sensevy, G. (2001). Théories de l'action et action du professeur. In J.-M. Baudouin \& J. Friedrich (Ed.), Théories de l'action et éducation (pp. 203-224). Paris, Bruxelles: De Boeck Université, Collection Raisons Educatives.

Sensevy, G., Mercier, A. \& Schubauer-Leoni, M.L. (2000). Vers un modèle de l'action didactique du professeur. A propos de la course à 20. Recherches en Didactique des Mathématiques, 20 (3), 263-304.

Thévenaz-Christen, T. (2002a). La tâche de l'enseignant dans l'explication d'une règle de jeu. Actes du 8ème congrès international de la DFLM, (Neuchâtel, 26-28 septembre 2001). [CD-ROM]. Neuchâtel: DFLM, INRP.

Thévenaz-Christen, T. (2002b). Milieu didactique et travail de l'élève dans une interaction maître-élève: apprendre à expliquer une règle de jeu à l'école enfantine genevoise. In $\mathrm{P}$. Venturini, C. Amade-Escot \& A. Terrisse (Ed.), Etudes des pratiques effectives: l'approche des didactiques, (pp.25-46). Grenoble: La pensée Sauvage, Collection Travaux et thèses de didactique.

\section{Vergleichende Studie über eine Aufgabe, realisiert mit Kindern im Alter von 4 Jahren in Krippe und Kindergarten in Genf}

\section{Zusammenfassung}

Dieser Beitrag aus dem Bereich der vergleichenden Didaktik basiert auf dem Konzept des contrat didactique und dessen topogenetischen, chronogenetischen und mesogenetischen Dimensionen. Diese theoretischen Instrumente werden üblicherweise angewandt, um das Verhalten von Kindern im Schulalter zu verstehen. In dieser Studie werden sie versuchsweise zur Analyse des Verhaltens von Vorschulkindern angewendet. Die Studie basiert auf einer von verschiedenen Lehrern entwickelten Aufgabe, welche Kindern im Alter von 4 Jahren zur Lösung gegeben werden soll. Die Aufgabe wird auf zwei Ebenen angewandt: im schulischen Kontext (Kindergarten) und vorschulischen Kontext (Krippe). Das Ziel ist der Vergleich der Resultate der Aufgabe in zwei verschiedenen Institutionen, welche aber beide Kinder im Alter von 4 Jahren aufnehmen. Die Analyse konzentriert sich auf eine Problemstellung, welche bereits im Voraus festgelegt wurde: die Problemlösungsstrategie der erwachsenen Personen (Lehrerin versus Erzieherin), mit denen sie die anstehenden Probleme angehen. Es zeigte sich, dass die Problemlösungsstrategien sich als unterschiedlich erwiesen und jeweils dem spezifischen didaktischen Kontext angepasst wurde. 


\section{Un'analisi comparata di un compito "scolastico" realizzato con bambini di 4 anni alla scuola dell'infanzia e all'asilo nido a Ginevra}

\section{Riassunto}

Questo contributo s' iscrive nel campo della didattica comparata ed è organizzato attorno al concetto di contratto didattico e delle sue dimensioni topogenetiche, cronogenetiche e mesogenetiche. Questi strumenti teorici, solitamente adoperati per comprendere le pratiche scolastiche, vengono ripresi, a titolo sperimentale, per l'analisi delle pratiche nel contesto prescolastico. La ricerca prende spunto da un compito creato da un gruppo di docenti della scuola dell'infanzia con lo scopo di proporla a degli alunni di 4 anni. Il compito è stato realizzato ed analizzato nei due ambiti: in quello della scuola dell'infanzia ed in quello di un asilo nido. Lo scopo è quindi di confrontare la realizzazione di un compito preciso in due istituzioni distinte, che accolgono, in entrambi i casi, bambini di 4 anni. L'analisi si concentra su una difficoltà specifica del compito, rilevata a priori. Le tecniche alle quali fanno appello le due professioniste (docente ed educatrice) per gestire questa difficoltà sono distinte anche se mirano a finalità fondamentalmente simili.

\section{Comparative analysis of a task performed by preschool and daycare center 4-year-old children.}

\section{Summary}

This paper falls into the field of comparative didactics and is concentrated around the concept of didactic contract and its topogenetic, chronogenetic and mesogenetic dimensions. These theoretical instruments, usually used to understand school praxis, are experimentally used here in order to analyze practices in a preschool context. The study is based on a task conceptualized by a group of teachers and intended for 4-year-old children. The task is car-ried out and studied in two contexts: pre-school and daycare. The aim is thus the comparison of the task within these two distinct institutions, both with 4-year-old children. The analysis focuses on a specific difficulty of the task, identified through an a priori analysis. The techni-cal approaches of the professionals (teachers $v$ s. educators) differ, although their funda-mental outcomes are the same. 
Annexe 1

\section{La tâche 1E:}

La tâche inventée par les enseignantes se présente de la manière suivante. Elle apparaît ici sous la forme originale telle que nous l'avons reçue.

\section{Activité $1 \mathrm{E}$}

Matériel: $\quad$ Plots de différentes couleurs (6 à 8 plots)

3.4 couleurs

1 forme

Nombre enfants: 4 dont 1 meneur

Pré-requis: Les plots ont été présentés et manipulés avant cette leçon

Objectif: $\quad$ Amener l'enfant à prendre conscience de la position des objets les uns par rapport aux autres

Déroulement: les enfants sont alignés, le meneur est devant les autres et leur tourne le dos (cf. schéma)

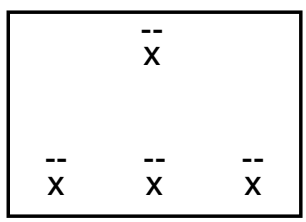

Vérifier qu'ils aient tous les mêmes plots

Le meneur construit son château en décrivant ce qu'il fait; les autres le font en même temps

Discussion, mise en commun

Consignes: $\quad$ Construis ton château en fonction des indications du meneur 